\title{
An integrative modular approach to systematically predict
} gene-phenotype associations

\author{
Michael R Mehan ${ }^{1}$, Juan Nunez-Iglesias ${ }^{1}$, Chao Dai ${ }^{2,1}$, Michael S Waterman ${ }^{1}$ \\ and Xianghong Jasmine Zhou*1
}

Addresses: ${ }^{1}$ Program in Computational Biology, Department of Biological Sciences, University of Southern California, Los Angeles CA 90089, USA and ${ }^{2}$ School of Computer, Wuhan University, Wuhan 430079, PR China

E-mail: Michael R Mehan - rielmeha@usc.edu; Juan Nunez-Iglesias - nunezigl@usc.edu; Chao Dai - chaodai@usc.edu;

Michael S Waterman - msw@usc.edu; Xianghong Jasmine Zhou* - xjzhou@usc.edu

${ }^{*}$ Corresponding author

from The Eighth Asia Pacific Bioinformatics Conference (APBC 2010)

Bangalore, India 18-2I January 2010

Published: 18 January 2010

BMC Bioinformatics 2010, II(SuppI I):S62 doi: 10.1186/I47I-2I05-II-SI-S62

This article is available from: http://www.biomedcentral.com/I47I-2I05/II/SI/S62

(C) 2010 Mehan et al; licensee BioMed Central Ltd.

This is an open access article distributed under the terms of the Creative Commons Attribution License (http://creativecommons.org/licenses/by/2.0), which permits unrestricted use, distribution, and reproduction in any medium, provided the original work is properly cited.

\begin{abstract}
Background: Complex human diseases are often caused by multiple mutations, each of which contributes only a minor effect to the disease phenotype. To study the basis for these complex phenotypes, we developed a network-based approach to identify coexpression modules specifically activated in particular phenotypes. We integrated these modules, protein-protein interaction data, Gene Ontology annotations, and our database of gene-phenotype associations derived from literature to predict novel human gene-phenotype associations. Our systematic predictions provide us with the opportunity to perform a global analysis of human gene pleiotropy and its underlying regulatory mechanisms.
\end{abstract}

Results: We applied this method to 338 microarray datasets, covering 178 phenotype classes, and identified 193, I 45 phenotype-specific coexpression modules. We trained random forest classifiers for each phenotype and predicted a total of 6,558 gene-phenotype associations. We showed that $40.9 \%$ genes are pleiotropic, highlighting that pleiotropy is more prevalent than previously expected. We collected 77 ChIP-chip datasets studying 69 transcription factors binding over 16,000 targets under various phenotypic conditions. Utilizing this unique data source, we confirmed that dynamic transcriptional regulation is an important force driving the formation of phenotype specific gene modules.

Conclusion: We created a genome-wide gene to phenotype mapping that has many potential implications, including providing potential new drug targets and uncovering the basis for human disease phenotypes. Our analysis of these phenotype-specific coexpression modules reveals a high prevalence of gene pleiotropy, and suggests that phenotype-specific transcription factor binding may contribute to phenotypic diversity. All resources from our study are made freely available on our online Phenotype Prediction Database [I]. 


\section{Background}

A major goal of modern genetics is to determine which genes are associated with which human phenotypes. Over the course of the last few decades, studies uncovering the basis for Mendelian diseases have been extremely successful, typically identifying causal mutations in single genes [2]. However, most human phenotypes, e.g. complex diseases such as cancer or neurological diseases, are controlled by multiple genes, each of which has a minor contribution to the disease phenotype [3]. Therefore, to effectively identify genes that are related to complex phenotypes, the approach must consider groups of genes rather than studying genes in isolation. Numerous methods have been developed for identifying gene modules from protein-protein interaction (PPI) networks [4], metabolic networks [5], or transcription-regulatory networks [6], however these network data are often lack human phenotype-specific information.

In this study, we identified gene modules that are specifically coexpressed in datasets that study particular human phenotypes. Identifying phenotype-specific modules in human is far more difficult than in model organisms because the phenotypes can only be observed rather than working directly with the biological pathways that define them. Ideally, these biological pathways should be reverse-engineered from data taken from individuals that display particular phenotypes. Public repositories of microarray data are a valuable resource for this type of analysis because they contains hundreds of well annotated expression datasets that span a wide variety of phenotypic conditions. It is known that identifying co-expression modules frequently occurring across multiple microarray datasets significantly enhances the signal to noise ratio [7-9]. Here, we identified co-expression modules that are present recurrently and specifically in datasets of one phenotype by using the remaining datasets as a background.

Using these phenotype-specific coexpression modules, we performed a systematic prediction of gene-phenotype associations by integrating three data sources: previously known associations derived from literature text mining, Gene Ontology annotations, and protein-protein interactions. A previous study designed an approach to identify gene modules in human PPI networks and used them to predict novel gene-phenotype associations [10]. Our approach differs from this in that it integrates hundreds of microarray datasets in parallel to identify modules, and then superimposes protein-protein interactions as well as phenotype and functional annotations to make predictions.
The systematic annotation of gene-phenotype associations provides us with the opportunity to perform the first global analysis of gene pleiotropy in human. Gene pleiotropy has the potential to explain the vast human phenotypic diversity, considering that the number of human genes is far fewer than originally anticipated [11]. Several large scale studies of pleiotropy have been performed on model organisms such as yeast $[12,13]$ and C. elegans [14]. In humans however, pleiotropy is often only recognized following the in-depth analysis of a single gene or gene family [15-17]. To our knowledge, no comprehensive determination or prediction of which genes exhibit pleiotropic behavior throughout the entire human genome has been previously performed. Based on our modular approach, we have defined a novel concept, modular pleiotropy, as the pleiotropic behavior of genes resulting from their presence in their modules, as we have shown that changes in module membership can define a gene's pleiotropic behavior. To further understand the underlying mechanisms of phenotypic diversity, we utilized the rapid accumulation of ChIP-chip datasets, measured under various phenotypic conditions, and tested whether the phenotype specificity of our modules, as well as specific instances pleiotropy, could be attributed to dynamic phenotype-specific gene regulation.

By integrating 338 human microarray datasets representing 283 phenotypes, we have identified 193,145 phenotype-specific modules. We subsequently predicted 6,558 novel gene-phenotype associations covering 3,183 genes, and showed that $40.9 \%$ of genes are associated with multiple phenotypes, and can thus be considered pleiotropic. We collected 77 Chip-chip datasets, annotated them with matching phenotypes, and confirmed that dynamic transcriptional regulation is an important force driving the formation of phenotype-specific modules. Our module-based approach has the advantage of not only predicting pleiotropy, but also suggesting how a gene is pleiotropic, exerting different phenotypic functions in different transcriptional and regulatory contexts.

We have provided all the data from our study, including the gene phenotype database we constructed via text mining, our phenotype-specific modules, and our novel phenotype predictions, in our online Phenotype Prediction Database [1].

\section{Results and discussion}

Systematic annotation of gene-phenotype association

Our approach identified phenotype-specific modules preferentially coexpressed in microarray datasets that 
study particular phenotypes, and we used these modules to perform systematic phenotype prediction, study gene pleitropy, and integrated phenotype-specific transcription factor binding data to build dynamic regulatory networks (Figure 1). We collected 338 human microarray datasets from the NCBI Gene Expression Omnibus (GEO) [18]. By mapping the dataset annotations onto UMLS [19] phenotype terms we obtained 283 phenotype classes each of which contained at least 3 microarray datasets. For the purposes of this paper, we employ a broad interpretation of the term phenotype, which includes diseases, tissues, and cell types.

We previously designed a Multiple Objective Simulated Annealing (MOSA) algorithm that robustly identifies groups of genes that are preferentially coexpressed in datasets of a specific phenotype class [20]. The simulated annealing procedure was designed to optimize four characteristics of phenotype-specific coexpression modules: size, density, specificity for the phenotype datasets, and a summary statistic of module density which we term density differential. The goal of this approach was to identify genes modules that exhibit a coexpression signature which is specific to the phenotype in which they were identified, and therefore are likely to represent processes inherent to the phenotype.

Applying this approach, we identified 193,145 phenotype-specific coexpression modules that met our criteria of a minimum size of 7 , minimum density of 0.66 , and a minimum phenotypic enrichment $p$-value of less than 0.01 after FDR correction. These modules spanned 178 phenotypes and had an average size of 13.7. A more detailed description of this algorithm is outlined in the methods section.

In this study, starting with the phenotype-specific modules, we predicted gene phenotype associations by additionally incorporating the following three data sources: our database of gene-phenotype associations obtained from text-mining of the Gene Reference Into Function (GeneRIF) resource, the Gene Ontology database, and protein-protein interaction data (details in methods).

We trained a different random forest classifier for the 52 phenotypes for which the phenotype-specific coexpression modules contained genes with GeneRIF entries with the same phenotype. Each classifier therefore depended on the the set of genes that were known to be associated with the training phenotype via GeneRIF text mining, to which we refer to as $G_{p}$. Our model contained 5 predictor variables, and contained one observation for each gene in the phenotype-specific modules. If a gene appeared in more than one module, the predictor variables were averaged across the modules.

Given a previously unannotated gene $g_{i}$, which is a member of module $m_{p j}$ (the $j^{\text {th }}$ module specific to phenotype $p$ ), we predicted whether $g_{i}$ is associated with phenotype $p$ by considering the following predictive features: i) the enrichment of the members of $m_{p j}$ for genes in $G_{p}$; ii) the degree of GO annotation similarity between $g_{i}$ and $m_{p j} \cap G_{p}$; iii) and the number of proteinprotein interactions between $g_{i}$ and $m_{p j} \cap G_{p}$. The first predictor variable was the negative $\log p$-value of the hypergeometric test for enrichment of genes in the module for genes in $G_{p}$. The second predictor variable was derived from protein-protein interaction (PPI) data. The PPI score was calculated by summing the total number of protein-protein interactions between the current gene and genes from $G_{p}$ in the same module. The remaining three predictor variables were derived from the three subtrees of GO (biological process, cellular component, and molecular function) as follows. For a pair of genes in the same module, the GO score for a subtree was calculated by first identifying all GO terms shared between the two genes. Once this set of distinct matching terms was established, the GO score was calculated by summing up the negative $\log p$-values of the significance of matches to genes in $G_{p}$.

These classifiers trained on the predictive features discussed above predicted 6,558 gene-phenotype associations covering 3,183 genes. For a cumulative recall (including all phenotypes) of at least 20\%, the precision of our predictions was approximately $65 \%$. As the stringency of the cutoff parameter increases the precision continues to climb above $80 \%$. This indicates that if we restrict the classifiers to a small number of predictions they become extremely accurate, which would be an ideal set to pursue in a clinical setting. The precisionrecall plot, which differs from a ROC plot by only plotting statistics related to positive predictions, for three classifiers compared to randomly generated modules is shown in Figure 2. Each of our predictions is scored based on the fraction of classification trees from the random forest that voted for the prediction. Table 1 shows the predictions with the best scores for the 10 highest scoring predictions, stratified by phenotype, along with supporting evidence from published papers and Gene Ontology. GeneRIF does not contain all published papers that discuss a gene's association with a phenotype, so the published papers listed in this table represent information not used by the classifier. The first prediction is ADRB3 for the phenotype "Urologic 


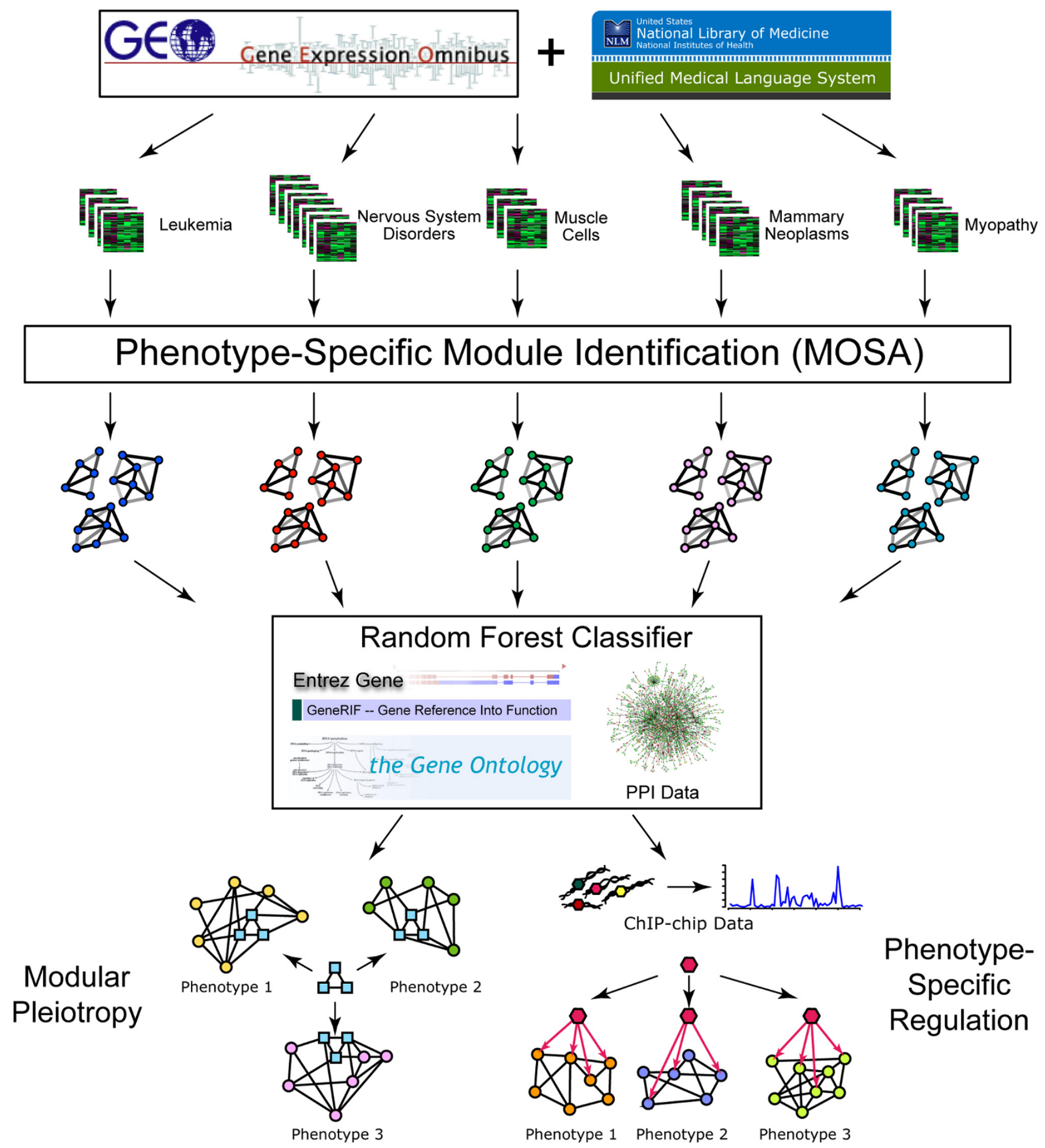

Figure I

A flow chart of our approach pipeline for each phenotype. We designed a multiple objective simulated annealing (MOSA) algorithm to identify phenotype-specific coexpression modules in microarray datasets. We incorporated additional data sources into a random forest classifier to make novel gene-phenotype predictions. These modules and predictions were used to study human pleiotropy and phenotype-specific transcription regulation. 

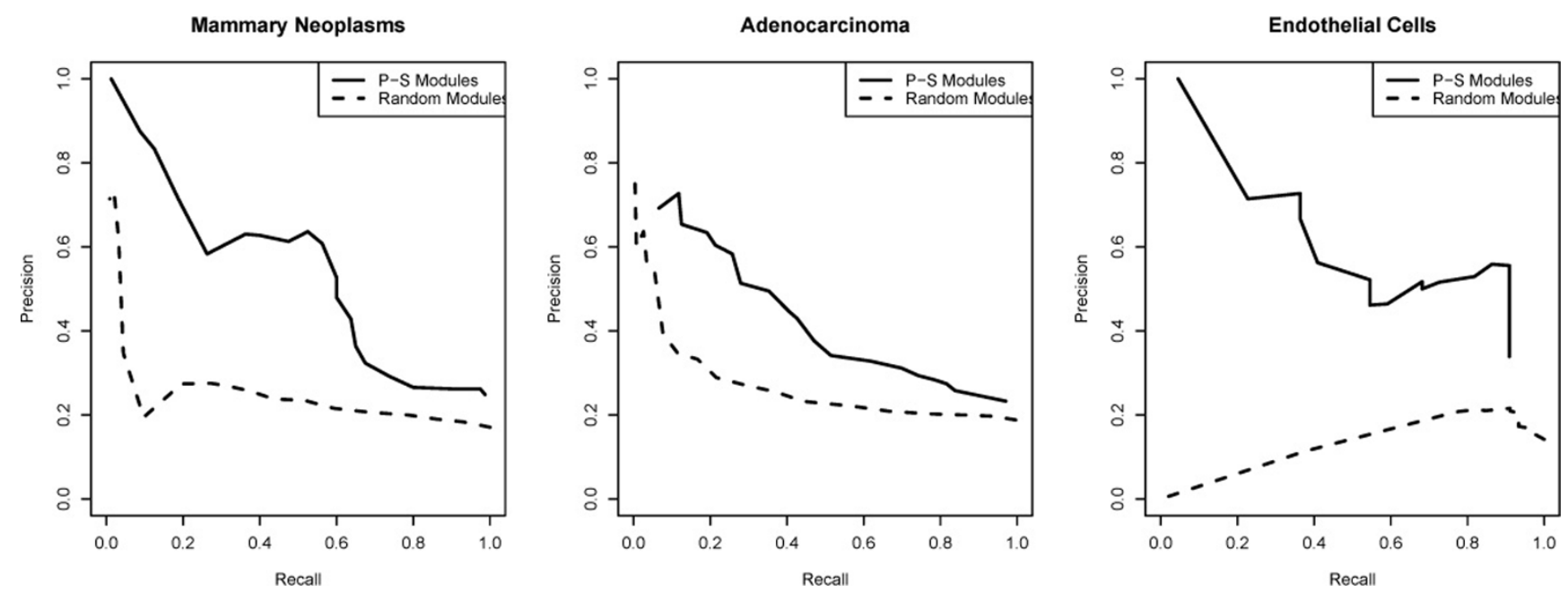

Figure 2

Random forest performance. Precision-recall plot depicting the training performance of the random forest classifiers for three phenotypes.

Table I: Highest confidence gene-phenotype association predictions stratified by phenotype

\begin{tabular}{|c|c|c|c|c|c|}
\hline Phenotype & Cutoff & Gene & Gene Description & PMID & Relevant GO Term \\
\hline Urologic Diseases & 1.00 & ADRB3 & adrenergic, beta-3-, receptor & $|83| 1486$ & \\
\hline Phagocytes & 1.00 & PML & promyelocytic leukemia & & myeloid cell differentiation \\
\hline Genital Neoplasms, Female & 1.00 & EXOI & exonuclease I & 15328369 & DNA repair \\
\hline Inflammation & 1.00 & CXCL2 & chemokine (C-X-C motif) ligand 2 & 12892904 & inflammatory response \\
\hline skin disorder & 0.99 & SC4MOL & sterol-C4-methyl oxidase-like & & \\
\hline Lymphocyte & 0.99 & FPRLI & formyl peptide receptor 2 & 15625007 & $\begin{array}{l}\text { G-protein coupled receptor protein } \\
\text { signaling pathway }\end{array}$ \\
\hline monocyte & 0.99 & TAP2 & $\begin{array}{l}\text { transporter } 2 \text {, ATP-binding cassette, } \\
\text { sub-family B (MDR/TAP) }\end{array}$ & 12234057 & immune response \\
\hline Bone Marrow Cells & 0.99 & CXCLII & chemokine (C-X-C motif) ligand II & 15102366 & immune response \\
\hline Epithelium & 0.98 & ITGB4 & integrin, beta 4 & 15194479 & cell adhesion \\
\hline Neoplasms & 0.98 & HMHAI & histocompatibility (minor) HA-I & 14502255 & intracellular signaling cascade \\
\hline
\end{tabular}

Each phenotype is listed with the predicted gene, the gene's description, and the proportion of decision trees from the random forest classifier that predicted its association to the phenotype. The final two columns provide supporting evidence in the form of a PubMed ID for a published work or a Gene Ontology annotation that is consistent with the phenotype.

Diseases." The potential for this gene to be involved in urologic diseases is supported by its presence in the human urinary bladder urothelium, as well as its regulation of urinary function [21]. Five of the predictions in this table are for phenotypes related to the immune system, such as "Phagocytes," "Inflammation," "Lymphocyte," "monocyte," and "Bone Marrow Cells." Many of these predictions are supported by both literature and their GO annotations which include myeloid cell differentiation, inflammatory response, and immune response. Two other interesting predictions are the chemokine genes CXCL11 and CXCL2 for the phenotypes "Bone Marrow Cells" and "Inflammation" respectively. Chemokines are master controllers of the migration of leukocytes, which originate in bone marrow and directly effect many functions related to the immune system including inflammation. The remaining predictions shown in the table are related to cancer and epithelial tissue related phenotypes.

To provide a more comprehensive analysis of our predictions, rather than just highlighting the highest scoring predictions, we performed functional enrichment analysis on each predicted set of associated genes for each phenotype. This analysis revealed that the majority of phenotype enriched biological processes are consistent with the functionality of the phenotype, which supports the quality of our predictions. For example, in the phenotype "Squamous cell carcinoma," our predicted genes are enriched for GO annotations related to skin cancer such as DNA replication, keratinization, and epidermis development. GO annotations are 
also consistent with the phenotypes for tissues. The phenotype "Brain" is enriched for synaptic transmission and monovalent inorganic cation transport. We highlight additional examples of significantly (FDR $p$-value < 0.01 ) overrepresented GO terms within predicted gene sets for phenotypes in Table 2.

\section{The prevalence of gene pleiotropy}

Our large-scale gene-phenotype association prediction provided us with a unique opportunity to systematically study gene pleiotropy. The simplest definition of pleiotropy, a gene being annotated with multiple UMLS phenotype terms, is inadequate here. For example, TAL1 is annotated with both "leukemia" and "Immunoproliferative Disorders," but these do not constitute two distinct phenotype associations because one is simply a more general phenotype than the other. Other groups have employed phenotype distance metrics and declared two phenotypes different when their distance exceeded a certain threshold $[10,22]$. However, this type of method relies on the selection of an arbitrary threshold, which can significantly affect the results. Here, we defined pleiotropy using the structure of the parental links within the UMLS phenotype ontology. We considered a gene pleiotropic if it was annotated with at least two phenotypes, neither of which is a descendant of the other.

Applying this definition of pleiotropy to only the disease phenotypes revealed that $40.9 \%$ of the 8,504 genes in our study are associated with at least one pair of distinct phenotypes. It should be noted that this percentage is likely to be an over estimate of pleiotropic genes, since
UMLS text mining of GeneRIF terms can lead to incorrect annotations and therefore pleiotropy. The addition of our phenotype predictions resulted in 607 novel pleiotropic genes. Furthermore, 725 genes that were previously pleiotropic were annotated with a new phenotype distinct from all previous annotations, resulting in a new case of pleiotropy.

Pleiotropy can be determined and observed in a variety of ways. In this study, we predicted whether a particular gene exhibits pleiotropic behavior based upon its membership in phenotype-specific coexpression modules. We therefore term this type of pleiotropy modular pleiotropy, in which a gene's pleiotropic behavior is determined by module membership. An example that illustrates this phenomenon is depicted in Figure 3 involving two modules, one specific to "nervous system disorders" and the other to "Neoplasms, Glandular and Epithelial." These modules share six genes related to the extracellular matrix, the substrate upon which cells migrate, proliferate, and differentiate. It is not surprising to find these genes associated with epithelial cancer, as many studies have shown that the extracellular matrix is heavily involved in tumor progression and metastasis $[23,24]$. The extracellular matrix also plays a major role in the development and repair of the central nervous system, supporting its presence in both modules [25]. In each of the two overlapping modules, the remaining, unshared genes also include genes from the extracellular matrix and additional genes that are highly specific to the phenotypes in which the modules were discovered. These include the well known Alzheimer's susceptibility locus APOE, an actin gene differentially expressed in schizophrenia patients (ACTG1), a brain-specific tubulin

Table 2: Overrepresented GO terms in predicted genes. Seven of the UMLS phenotypes are highlighted, along with the number of phenotype-specific coexpression modules and number of novel predicted associated genes. The final column contains a subset of the overrepresented GO biological processes in the predicted genes that are consistent with the phenotype. The full table is available on our supplementary website

\begin{tabular}{|c|c|c|c|}
\hline Phenotype & Modules & Predictions & Over-represented GO annotations \\
\hline Adenocarcinoma & 2367 & 69 & $\begin{array}{l}\text { cell cycle process }(3.5 \mathrm{e}-06) \\
\text { DNA replication }(4.1 \mathrm{e}-06) \\
\text { cell cycle phase }(8.5 \mathrm{e}-06)\end{array}$ \\
\hline Bone Marrow Cells & 5390 & 421 & $\begin{array}{l}\text { immune response }(3.8 \mathrm{e}-06) \\
\text { immune system process }(7.2 \mathrm{e}-06) \\
\text { response to virus }(2.0 \mathrm{e}-05)\end{array}$ \\
\hline Brain & 8373 & 329 & $\begin{array}{l}\text { synaptic transmission }(3.8 \mathrm{e}-06) \\
\text { monovalent inorganic cation transport }(1.7 \mathrm{e}-05)\end{array}$ \\
\hline Connective and Soft Tissue Neoplasm & 1463 & 22 & $\begin{array}{l}\text { cell cycle process }(4.3 \mathrm{e}-14) \\
\text { cytoskeleton-dependent intracellular transport }(8.6 \mathrm{e}-07)\end{array}$ \\
\hline Musculoskeletal Diseases & 5421 & 324 & actin filament-based process $(8.2 \mathrm{e}-06)$ \\
\hline Squamous cell carcinoma & 1268 & 54 & $\begin{array}{l}\text { DNA replication }(2.5 \mathrm{e}-08) \\
\text { keratinization }(8.1 \mathrm{e}-05) \\
\text { epidermis development }(1.0 \mathrm{e}-04)\end{array}$ \\
\hline nervous system disorder & 8235 & 628 & $\begin{array}{l}\text { respiratory electron transport chain }(5.1 \mathrm{e}-07) \\
\text { acetyl-CoA catabolic process }(3.2 \mathrm{e}-05)\end{array}$ \\
\hline
\end{tabular}




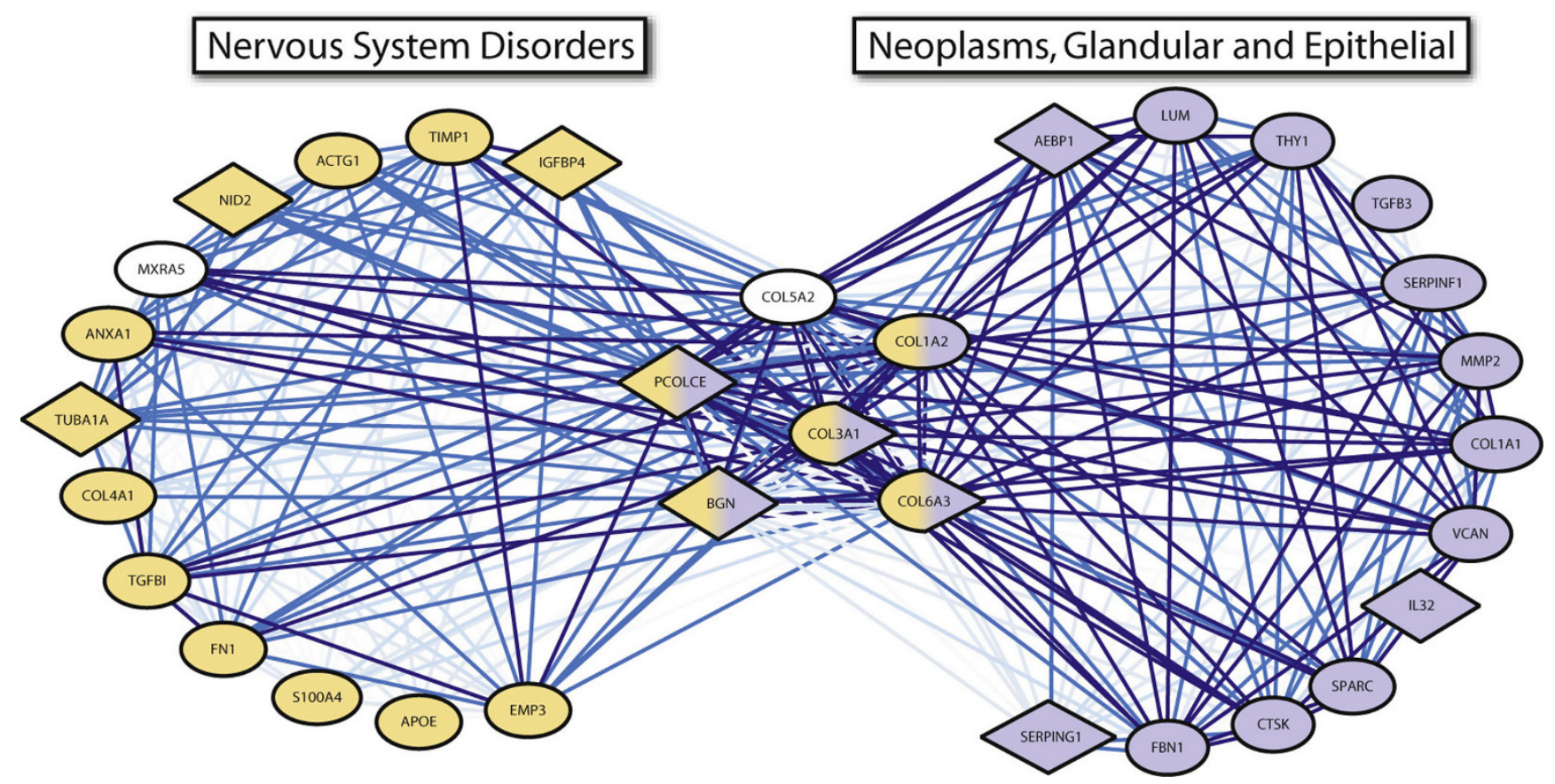

Figure 3

An example of modular pleiotropy with a pleiotropic component related to the extracellular matrix. The modules shown are specific to microarray datasets that study "nervous system disorders," and "Neoplasms, Glandular and Epithelial." The shaded elliptical nodes represent genes associated with the module phenotype (via GeneRIF text mining), whereas the shaded diamond nodes represent predictions made by our random forest classifier. The edge opacity indicates how often a pair of genes was coexpressed in the microarray datasets for which the module was coexpressed.

associated with behavioral defects (TUBA1A), and a tubulin polymerizer that serves as a neuroprotectant (S100A4). The neoplasms module also contains genes related to the extracellular matrix, and genes with known roles in neoplastic processes such as tumor progression, tumor necrosis induction, tumor suppression, and tumor invasion $[26,27]$.

We would also like to highlight an interesting prediction of pleiotropy for the gene BGN, which is present in both modules. This prediction is supported by the RefSeq entry for this gene, which states that it is thought to transfer growth factors between cells and that it may promote neuronal survival. Some additional evidence for BGN's activity in cancer was discovered by a study that showed BGN controls cell growth in pancreatic cancer cells [28]. Studies have demonstrated BGN's potential role in the human nervous system as well, as BGN is overexpressed in rats after brain injury and it sustained the survival of rat neocortical neurons in culture $[29,30]$.

The above example nicely illustrates the power of our classification method to make reliable phenotype predictions, and in particular to identify pleiotropy and its modular context.

\section{Phenotype-specific transcriptional regulation}

We hypothesized that regulatory networks are not static relationships between transcription factors and their target genes, but rather dynamic networks that vary to dictate different observed phenotypes, such as tissue types and diseases. Thanks to the accumulation of ChIPchip experiments, each of which provides genome-wide TF binding data derived under particular phenotypic conditions, we were able to test this hypothesis by determining whether TF binding detected under a phenotypic condition preferentially occurs in genes belonging to modules specific to the same phenotype. This concordance between or phenotype-specific coexpression modules and phenotype-specific binding from ChIP-chip data also serves to independently validate the phenotype-specificity of our modules.

We compiled 77 ChIP-chip datasets from the public repositories and also manually collected results from literature publications. These data include 69 TFs and 16,122 target genes, which can be found on our online Phenotype Prediction Database [1]. Applying our text mining procedure to these datasets resulted in a total of 208 phenotypes including diseases, tissues and cell types. Of these 208 phenotypes, we focused on the 97 
phenotypes that were also studied by the microarray datasets we had collected. This provided us with one of the most comprehensive collections of regulatory data available, with the phenotypic conditions that each dataset studied.

For each phenotype-specific module, we tested the member genes for enrichment of TF binding derived from ChIP-chip datasets that study related phenotypes. Out of the 97 ChIP-chip phenotypes, 43 exhibited a statistically significant preferential binding (Mann-Whitney test, FDR $<0.05)$, indicating that target genes from ChIP-chip data are more likely to form coexpression modules in microarray datasets whose phenotype annotations match those of the ChIP-chip experiment. The full table of $p$-values resulting from this analysis are available online on our Phenotype Prediction Database [1].

One example of a phenotype that exhibits preferential ChIP-chip binding is "Prostatic Neoplasms," which is studied by a single ChIP-chip dataset profiling androgen receptor binding. The phenotype-specific preferential binding is shown in Figure 4. The proportion of phenotype-specific modules for this phenotype that are significantly enriched for androgen receptor binding (hypergeometric $p$-value $<0.025$ ) is over 16 times higher than the modules specific to other phenotypes. This strong over representation of significant binding enrichment for our modules demonstrates the ability of our method to detect evidence of phenotype-specific TF binding, despite being provided only a small fraction of the complete transcriptional regulatory information related to this phenotype.

The second example in the figure is the more general phenotype "Malignant Neoplasms". The distribution of TF binding enrichment is more continuous for this example due to the larger number of related phenotypespecific modules and ChIP-chip datasets. As with the previous example, there is a higher relative frequency of significantly bound modules specific to phenotypes that match the phenotype of the TF binding. Also, the frequency of the most significant binding enrichment (hypergeometric $p$-value $<0.025$ ) within the distribution of the related phenotype-specific modules is more than twice as high as the frequency of all other enrichment $p$ values. This can be attributed to the much higher quantity of ChIP-chip data available for this phenotype, which allowed for more significant binding for our transcription modules. This result implies that as more ChIP-chip data becomes available, we would expect to see the significance of binding enrichment of our

Prostatic Neoplams

Malignant Neoplasms

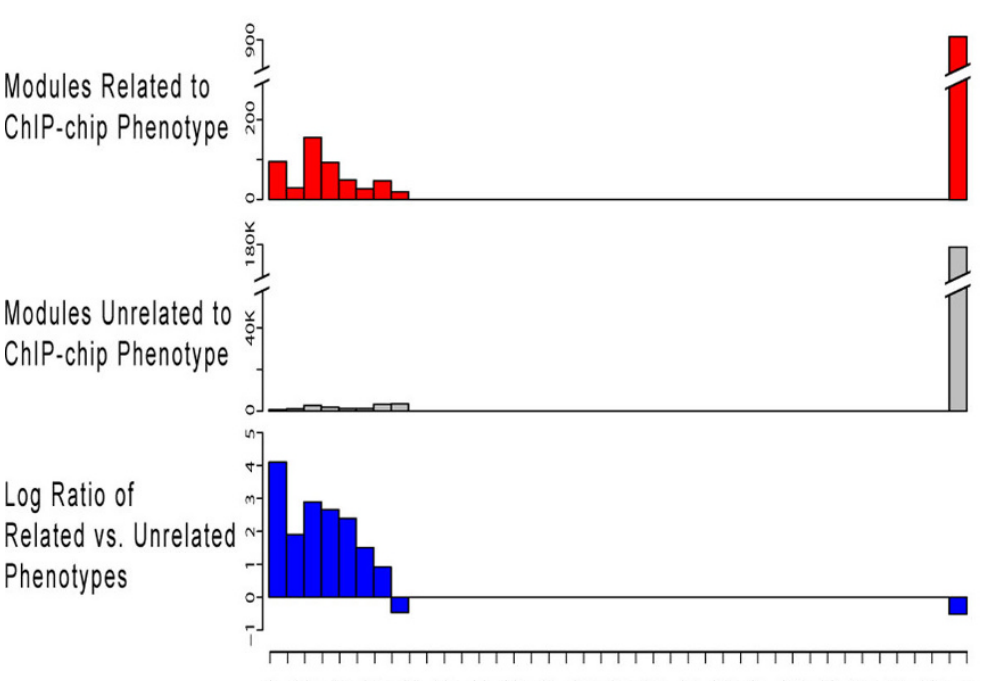

$\begin{array}{lllllllllllllllllllll}0 & 0.05 & 0.1 & 0.15 & 0.2 & 0.25 & 0.3 & 0.35 & 0.4 & 0.45 & 0.5 & 0.55 & 0.6 & 0.65 & 0.7 & 0.75 & 0.8 & 0.85 & 0.9 & 0.95 & 1\end{array}$

p-value

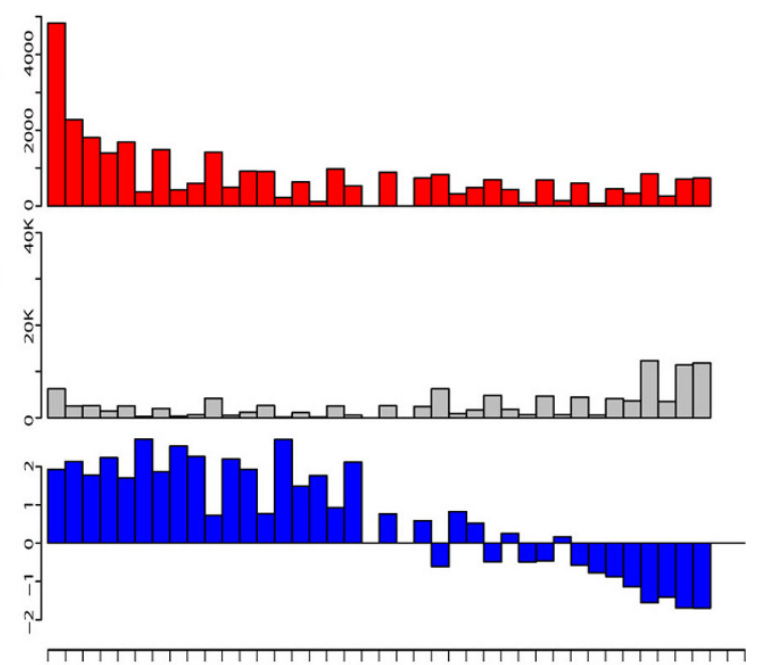

$\begin{array}{lllllllllllllllllllll}0 & 0.05 & 0.1 & 0.15 & 0.2 & 0.25 & 0.3 & 0.35 & 0.4 & 0.45 & 0.5 & 0.55 & 0.6 & 0.65 & 0.7 & 0.75 & 0.8 & 0.85 & 0.9 & 0.95 & 1\end{array}$

p-value

\section{Figure 4}

Distribution of ChIP-chip TF binding enrichment $p$-values for phenotype-specific coexpression modules. The distributions of ChIP-chip TF-binding enrichment $p$-values are shown for two ChIP-chip phenotypes: Prostatic Neoplasms and Malignant Neoplasms. The first and second rows of distributions correspond to those derived from microarray datasets with a phenotype related to the ChIP-chip data phenotype and those derived from unrelated phenotypes respectively. The final row displays the $\log _{2}$ ratio of the two distributions relative to their respective total sizes. 
phenotype-specific modules continue to increase. Although the complete set of phenotype-specific binding is not available, we were still able to construct portions of these dynamic transcriptional networks by combining phenotype-specific coexpression modules with TF binding from the same phenotypes. For example, Figure 5 shows two overlapping modules, each specific to different phenotypes, and each significantly enriched for phenotype-specific binding that matches its phenotype. The two modules are each preferentially coexpressed in datasets studying a specific phenotype: one "Squamous Cell Neoplasms" and the other "Immunoproliferative Disorders."

As with the previous example of pleiotropy, these two modules are phenotype specific and are enriched with genes related to each phenotype. The "Squamous Cell Neoplasms" module contains ten genes, five of which were previously annotated with the phenotype, and is also significantly enriched for GO terms consistent with cancer of squamous tissue: keratinocyte differentiation ( $p$-value $2.8 \mathrm{e}-9)$ and epidermis development ( $p$-value 5.1e-7). The seven gene "Immunoproliferative Disorders" module contains four genes that were annotated with the phenotype, as well as being enriched for GO annotations related to the immune system such as leukocyte chemotaxis ( $p$-value 5.1e-7) and defense response ( $p$-value 5.0e-6). The modules share two proinflammatory calcium binding genes, S100A9 and S100A12, that are implicated together in a number of diseases [31]. S100A9 was previously annotated with both phenotypes, making it an example of a pleiotropic gene confirmed by its presence in two coexpression modules.

Our analysis of phenotypic conditions of the ChIP-chip data revealed that each of these modules were significantly enriched for phenotype-specific TF binding. The

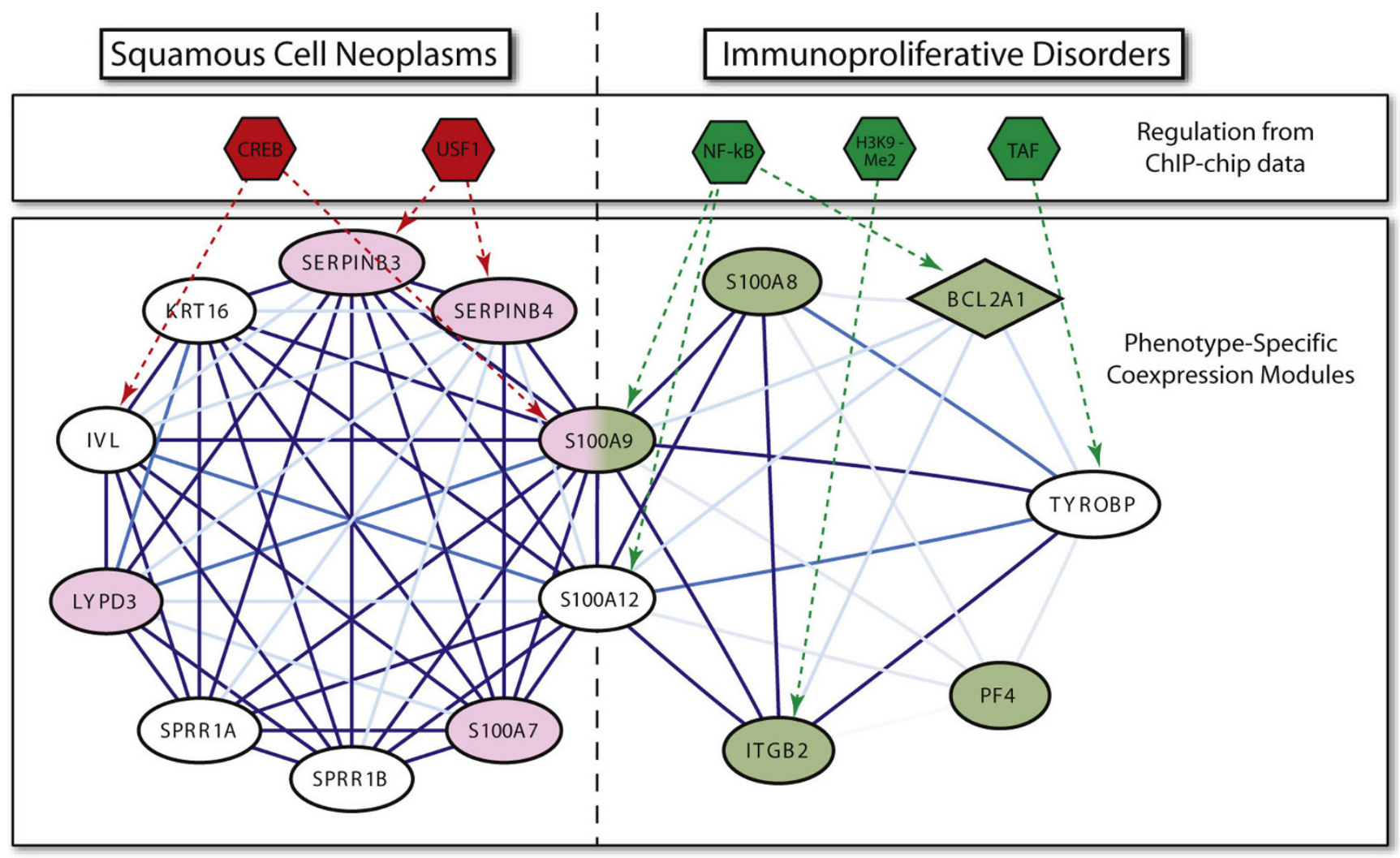

\section{Figure 5}

An example of modular pleiotropy with phenotype-specific regulatory data. The genes in the module on the left are specifically coexpressed in "Squamous Cell Neoplasms" microarray datasets, while those on the right are specifically coexpressed in "Immunoproliferative Disorders" microarray datasets. Two phenotype-specific modules containing genes with GeneRIF entries for the same phenotype (shaded). The edge opacity indicates how often a pair of genes was coexpressed in the microarray datasets for which the module was coexpressed. The opacity the edges indicates the degree of coexpression. The transcription factor binding, derived from ChIP-chip datasets that studied phenotypes related to the microarray datasets, is shown with dotted arrows. 
binding for the "Squamous Cell Neoplasms" module was derived from 18 ChIP-chip datasets that study the related phenotype "Epithelial Cells." The module is significantly enriched for the USF1 transcription factor ( $p$-value 0.02) which binds SERPINB3 and SERPINB4 in epithelial cells, as well as marginally enriched for CREB ( $p$-value 0.06) which binds IVL and S100A9.

The binding for the "Immunoproliferative Disorders" module was derived from datasets that study "Myeloid Cells," which was studied by 16 datasets. The majority of the binding was by the transcription factor NF- $k \mathrm{~B}$, which in myeloid cells binds three genes ( $p$-value 0.0006) and is known to play a role in regulation of the immune system as well as types of cancer [32]. This included both shared genes (S100A9, S100A12), and a gene that performs cytoprotective and anti-apoptotic roles in neutrophils (BCL2A1) [33]. This concordance between microarray expression data and regulatory ChIP-chip data demonstrates that our approach of finding phenotype-specific transcription modules can not only identify gene-phenotype relationships, but that it can be combined with TF binding data to further explain the molecular mechanisms and regulatory pathways underlying phenotypes and pleiotropy.

\section{Conclusion}

We have developed a method to determine the phenotypic effects of particular genes in humans, where phenotype prediction is far more difficult than in model organisms. This difficulty is largely due to our inability to study the underlying biological pathways directly, for example by inducing mutations or otherwise perturbing phenotype-related pathways. Our approach circumvents this limitation because the identified gene modules are preferentially active in datasets that study a particular phenotype, and thus are likely to represent phenotypespecific pathways. Using public resources that provide phenotype data, such as UMLS and GeneRIF, we made novel predictions that we believe will be useful in a clinical setting, further our understanding of disease pathways, and serve as potential drug targets.

In addition, we propose that regulatory networks should be viewed as dynamic networks with topology dependent upon the phenotypic conditions that they model. Since ChIP-chip experiments examine binding data under specific phenotypic conditions, their data provide a static snapshot of this dynamic regulatory network. Our results support this hypothesis by demonstrating preferential binding of transcription factors to coexpression modules in similar phenotypes.

Our pleiotropy analysis, complemented by our phenotype predictions, indicates that pleiotropy in humans may be a more widespread phenomenon than previously thought. Further study of how coexpression partners change in different tissues and the role phenotypespecific transcriptional regulation plays in shaping these changes will provide deeper insight into the underlying cause of phenotypic diversity as well as genetic pleiotropy.

\section{Methods}

Data sources

The microarray datasets were downloaded from NCBI GEO, and were from Affymetrix human platforms U95, U133, U133A 2.0, and U133 Plus 2.0. All datasets from these platforms with at least eight experimental samples were included, for a total of 338 datasets, which are included in the online Phenotype Database [1]. We only included the 8,504 genes that were present in all datasets and had at least one probe that mapped uniquely to it. We annotated each of the datasets with phenotype terms by mining the titles and MeSH terms of the associated papers with the UMLS MMTx tool, following the approach by Butte and Kohane [34].

\section{Creation of a database of GeneRIF gene-phenotype associations}

To objectively assign individual genes to the phenotypes with which they are known to be associated, we used the UMLS MetaMap Transfer tool to identify phenotypic terms from the UMLS Metathesaurus that were present in the raw text for each gene within Gene Reference Into Function (GeneRIF). GeneRIF submissions often contain concise information about the function of the gene, or whether it is known to be associated with any disease phenotypes. Therefore, by applying the UMLS MetaMap Transfer tool, we created a database of gene-phenotype associations derived from literature sources. Each association in the database was traced back to the root of the tree defined by the parental phenotypes described in the next section.

\section{Phenotype relationships}

For the Phenotype-Prediction and ChIP-chip analysis we defined a set of related phenotypes for each phenotype using the relationships provided by the UMLS Metathesaurus. The related phenotypes were defined by immediate neighbors using the set of relationships $\{R B, R L, R N$, $\mathrm{RO}, \mathrm{RQ}, \mathrm{RU}, \mathrm{SY}\}$. The relationships used represent different types of similarity as well as whether two phenotypes are simply synonymous. The parental phenotypes were defined using the "PAR" relationships from the UMLS Metathesaurus. For a given phenotype, all phenotypes present in the trace to the root of the UMLS graph defined by these "PAR" edges were considered parental phenotypes. 


\section{Gene module detection}

Our procedure is explained in detail in our previous paper [20]. For completeness, we present an overview here. The goal of the algorithm we used is to find sets of genes that are significantly coexpressed specifically in datasets related to particular phenotypes. For each of the 338 datasets in our study, we constructed an unweighted coexpression network composed of the top 150,000 correlated genes. We designed a multi-objective simulated annealing algorithm, with four objective functions designed to maximize the size, absolute density, relative density (to non-phenotype datasets), and specificity of the coexpression module for the phenotype datasets. The purpose of the relative density, or density differential, was to reward subtle changes in module density that would not be detected by the phenotype-specificity objective function. The objective functions we designed are outlined below.

$$
\begin{aligned}
& f_{\text {size }}(x)=\exp \left\{-\alpha\left(\frac{|x|}{\gamma}-o_{s}\right)\right\} \\
& f_{\text {dens }}(x)=\exp \left\{-\alpha\left(\min _{i \in \mathcal{G}_{\mathcal{A}}}\left(\delta_{i}(x)\right)-o_{\delta}\right)\right\} \\
& f_{\text {spec }}(x)=\log \left(\mathbb{P}\left(Y \geq\left|\mathcal{G}_{\mathcal{A}} \cap \mathcal{G}_{\mathcal{P}}\right|\right)\right) \\
& f_{\text {diff }}(x)=\left(\frac{1}{\left|\mathcal{G}_{\mathcal{P}}^{c}\right|} \sum_{i \in \mathcal{G}_{\mathcal{P}}^{c}} \delta_{i}(x)+\frac{1}{\left|\mathcal{G}_{\mathcal{P}}\right|} \sum_{i \in \mathcal{G}_{\mathcal{P}}} \delta_{i}(x)\right)
\end{aligned}
$$

where

$\mathcal{G}_{\mathcal{P}}$ is the set of datasets annotated with the current phenotype,

$\mathcal{G}_{\mathcal{A}}$ is the set of datasets in which the gene cluster is dense,

and $Y \sim$ hypergeometric $\left(\left|\mathcal{G}_{\mathcal{A}}\right|,\left|\mathcal{G}_{\mathcal{P}}\right|,\left|\mathcal{G}_{\mathcal{P}}^{c}\right|\right)$.

After completion of the simulated annealing procedure, we performed post filtering to remove low-quality modules. Our filtering criteria for each module was a minimum size of 7 , minimum density of 0.66 , and a minimum phenotypic enrichment $p$-value of less than 0.01 after FDR correction. These modules formed the basis for the phenotype prediction presented in this paper.

\section{Random forest training}

The random forest classifier was trained using 500 trees and a maximum terminal node size of 10 . The crossvalidation statistics reported were calculated using the Out Of Bag (OOB) errors provided by the training process. For each classifier, the positive training set was composed of all genes that contained a GeneRIF entry that mentioned the training phenotype. The negative set is more difficult to define, since genes that are actually associated with a particular phenotype may not be labeled as such due to imperfect text processing or missing GeneRIF annotations. Therefore, to minimize the error of our classifier we used the phenotype relationships defined by the UMLS to define our negative training set. We only included genes in the negative set that contained GeneRIF entries that were neither related nor parental to the training phenotype (see Section Phenotype relationships for more detail). The remaining genes, which included the related and parental genes, and those with no GeneRIF entries, were excluded from the training procedure and were used for prediction after the training of each phenotype classifier. The final predictions reported for each phenotype were the positive predicted genes from our training, as well as all positively predicted genes from our prediction set.

\section{ChIP-chip phenotype specificity}

The phenotypes studied by each ChIP-chip dataset was determined by performed text mining on the datasets' title, description, MeSH headings, and associated publications' title and abstract using the UMLS MMTx text mining tools. Given a single phenotype present in both ChIP-chip data and microarray data, we defined two sets of coexpression clusters: one derived from phenotypes related to the current phenotype, and the remaining modules unrelated to the current phenotype. We performed a Mann-Whitney test on the hypergeometric test $p$-values for TF enrichment in modules from related phenotypes versus the rest of the modules, to determine whether transcription factors from the phenotype ChIPchip experiments exhibited significant preferential binding.

\section{Competing interests}

The authors declare that they have no competing interests.

\section{Authors' contributions}

MM, JNE, CD, MSW, and XJZ designed the study and drafted the manuscript. MM implemented the simulated annealing algorithm, and performed the phenotype prediction and the subsequent analyses. JNE contributed to the analyses. CD collected and analyzed the ChIP-chip data. XJZ supervised the project. All authors read and approved the final manuscript.

\section{Acknowledgements}

This study was supported by the NIH grants R0IGM074I63, R2IAG03 I723, P5OHG002790, and the NSF grant 0747475. 
This article has been published as part of BMC Bioinformatics Volume II Supplement I, 2010: Selected articles from the Eighth Asia-Pacific Bioinformatics Conference (APBC 20I0). The full contents of the supplement are available online at http://www.biomedcentral.com/I47I$2105 / \mid I$ ? issue=SI

\section{References}

I. Phenotype Prediction Database. http://zhoulab.usc.edu/GenePhenoPred.

2. Wang W, Barratt B, Clayton D and Todd J: Genome-wide association studies: theoretical and practical concerns. Nature Reviews Genetics 2005.

3. Hirschhorn f and Daly M: Genome-wide association studies for common diseases and complex traits. Nature Reviews Genetics 2005, 6(2):95-108.

4. Han JD, Bertin N, Hao T, Goldberg DS, Berriz GF, Zhang LV, Dupuy D, Walhout AJ, Cusick ME, Roth FP and Vidal M: Evidence for dynamically organized modularity in the yeast proteinprotein interaction network. Nature 2004, 430:88-93.

5. Ravasz E, Somera A, Mongru D, Oltvai Z and Barabasi A: Hierarchical Organization of Modularity in Metabolic Networks.2002

6. Lee T, Rinaldi N, Robert F, Odom D, Bar-Joseph Z, Gerber G, Hannett N, Harbison C, Thompson C and Simon I, et al: Transcriptional Regulatory Networks in Saccharomyces cerevisiae. Science 2002, 298(5594):799-804.

7. Hu H, Yan X, Huang Y, Han J and Zhou XJ: Mining coherent dense subgraphs across massive biological networks for functional discovery. Bioinformatics 2005, 2I(Suppl I):i2 I3-2 I.

8. Huang $Y, \mathrm{Li} \mathrm{H}, \mathrm{Hu} \mathrm{H}$, Yan $X$, Waterman M, Huang $\mathrm{H}$ and Zhou $X$ Systematic discovery of functional modules and contextspecific functional annotation of human genome. Bioinformatics 2007, 23(I3):i222

9. Yan X, Mehan MR, Huang Y, Waterman MS, Yu PS and Zhou XJ: A graph-based approach to systematically reconstruct human transcriptional regulatory modules. Bioinformatics 2007 23(13):i577-86

10. Lage K, Karlberg EO, Størling ZM, Olason PI, Pedersen AG, Rigina O Hinsby AM, Tümer $Z$, Pociot $F$, Tommerup $N$, Moreau $Y$ and Brunak S: A human phenome-interactome network of protein complexes implicated in genetic disorders. Nat Biotechnol 2007, 25(3):309-16.

II. Pennisi E: Why do humans have so few genes? Science 2005 , 309(573I):80-80

12. He X and Zhang J: Toward a molecular understanding of pleiotropy. Genetics 2006, I73(4):|885-9|.

13. Dudley A, Janse D, Tanay A, Shamir R and Church G: A global view of pleiotropy and phenotypically derived gene function in yeast. Molecular systems biology 2005, I.

14. Piano F, Schetter A, Mangone M, Stein L and Kemphues K: RNAi analysis of genes expressed in the ovary of Caenorhabditis elegans. Current Biology 2000, 10(24):1619-1622.

15. Ridanpää $M$, van Eenennaam $H$, Pelin $K$, Chadwick R, Johnson $C$, Yuan B, vanVenrooij W, Pruijn G, Salmela R and Rockas $S$, et al: Mutations in the RNA component of RNase MRP cause a pleiotropic human disease, cartilage-hair hypoplasia. Cell 200I, 104(2): 195-203.

16. Badano JL, Mitsuma N, Beales PL and Katsanis N: The ciliopathies: an emerging class of human genetic disorders. Annu Rev Genomics Hum Genet 2006, 7:125-I48.

17. Sanchez-Cuenca J, Martin J, Pellicer A and Simon C: Cytokine pleiotropy and redundancy-gp 130 cytokines in human implantation. Immunology Today 1999, 20(2):57-59.

18. Barrett T, Troup DB, Wilhite SE, Ledoux P, Rudnev D, Evangelista C, Kim IF, Soboleva A, Tomashevsky M and Edgar R: NCBI GEO mining tens of millions of expression profiles-database and tools update. Nucleic Acids Res 2007, 35 Database: D760-5.

19. Bodenreider O: The Unified Medical Language System (UMLS): integrating biomedical terminology. Nucleic Acids Res 2004, 32 Database: D267-70.

20. Mehan MR, Nunez-Iglesias J, Kalakrishnan M, Waterman MS and Zhou X]: An Integrative Network Approach to Map the Transcriptome to the Phenome. J Comput Biol 2009.

21. Otsuka A, Shinbo $H$, Matsumoto $R$, Kurita $Y$ and Ozono S: Expression and functional role of beta-adrenoceptors in the human urinary bladder urothelium. Naunyn Schmiedebergs Arch Pharmacol 2008, 377:473-48I.
22. Ala U, Piro RM, Grassi E, Damasco C, Silengo L, Oti M, Provero P and Cunto FD: Prediction of human disease genes by humanmouse conserved coexpression analysis. PLoS Comput Biol 2008, 4(3): el 000043.

23. Gohji K, Hirano H, Okamoto M, Kitazawa S, Toyoshima M, Dong J, Katsuoka $Y$ and Nakajima M: Expression of three extracellular matrix degradative enzymes in bladder cancer. Int J Cancer (Pred Oncol) 200I, 95:295-30I

24. Tuxhorn I Ayala G, Smith M, Smith V, Dang T and Rowley D: Reactive Stroma in Human Prostate Cancer Induction of Myofibroblast Phenotype and Extracellular Matrix Remodeling I. Clinical Cancer Research 2002, 8(9):29|2-2923.

25. Rutka J, Apodaca G, Stern R and Rosenblum M: The extracellular matrix of the central and peripheral nervous systems: structure and function. J Neurosurg 1988, 69(2): 155-70.

26. Clark Cl and Sage $\mathrm{EH}$ : A prototypic matricellular protein in the tumor microenvironment-where there's SPARC, there's fire. J Cell Biochem 2008, 104:72I-732.

27. Dashevsky O, Varon D and Brill A: Platelet-derived microparticles promote invasiveness of prostate cancer cells via upregulation of MMP-2 production. Int J Cancer 2009, 124:1773-1777.

28. Chen $W$, Lenschow $W$, Tiede $K$, Fischer J, Kalthoff $H$ and Ungefroren H: Smad4/DPC4-dependent regulation of biglycan gene expression by transforming growth factor- $\beta$ in pancreatic tumor cells. Journal of Biological Chemistry 2002, 277(39): 36118-36128.

29. Stichel C, Kappler J, Junghans $U$, Koops $A$, Kresse $H$ and Müller $H$ : Differential expression of the small chondroitin/dermatan sulfate proteoglycans decorin and biglycan after injury of the adult rat brain. Brain Research 1995, 704(2):263-274.

30. Junghans $U$, Koops A, Westmeyer A, Kappler J, Meyer $H$ and Muller $\mathrm{H}$ : Purification of a meningeal cell-derived chondroitin sulphate proteoglycan with neurotrophic activity for brain neurons and its identification as biglycan. European Journal of Neuroscience 1995, 7(II):234I-2350.

31. Leach ST, Yang Z, Messina I, Song C, Geczy CL, Cunningham AM and Day AS: Serum and mucosal SI00 proteins, calprotectin (SIO0A8/SI00A9) and SIO0A I 2, are elevated at diagnosis in children with inflammatory bowel disease. Scand J Gastroenterol 2007, 42: $|32|-|33|$.

32. Karin M and Greten FR: NF-kappaB: linking inflammation and immunity to cancer development and progression. Nat Rev Immunol 2005, 5:749-759.

33. Schreiber J, Jenner RG, Murray HL, Gerber GK, Gifford DK and Young RA: Coordinated binding of NF-kappaB family members in the response of human cells to lipopolysaccharide. Proc Natl Acad Sci USA 2006, 103:5899-5904.

34. Butte $A J$ and Kohane IS: Creation and implications of a phenome-genome network. Nat Biotechnol 2006, 24:55-62.

Publish with BioMed Central and every scientist can read your work free of charge

"BioMed Central will be the most significant development for disseminating the results of biomedical research in our lifetime. "

Sir Paul Nurse, Cancer Research UK

Your research papers will be:

- available free of charge to the entire biomedical community

- peer reviewed and published immediately upon acceptance

- cited in PubMed and archived on PubMed Central

- yours - you keep the copyright
BioMedcentral 